\title{
Educación inclusiva en Ecuador: perspectiva de directores, familias y evaluadores
}

Inclusive Education in Ecuador: Perspective of Directors, Family and Evaluators

Artículo de investigación | Research article

Fecha de recepción: 26 de noviembre de 2018

Fecha de aceptación: 06 de agosto de 2019 Fecha de disponibilidad en línea: enero de 2021

doi: 10.11144/Javeriana.m14.eiep

Raúl TÁrRaga-Mínguez raul.tarraga@uv.es Universidad de VALenCIA, España https://orcid.org/0000-0002-4458-5763

(iD) ORCID

Ximena Vélez-Calvo xvelez@uazuay.edu.ec Universidad del AzUAY, ECUADOR https://orcid.org/0000-0002-4451-9547

(iD) ORCID

Pilar Sanz-Cervera pilar.sanz-cervera@uv.es Universidad de Valencia, España https://orcid.org/0000-0001-6919-6150
Gemma Pastor-Cerezuela gemma.pastor@uv.es UnIVERSIDAD de VALENCIA, EsPAÑa https://orcid.org/0000-0002-4992-4701

(iD) ORCID

María Inmaculada Fernández-Andrés (†) m.inmaculada.fernandez@uv.es Universidad de VALencia, EsPaña https://orcid.org/0000-0003-2808-0949

(iD) ORCID

(iD) ORCID

Para citar este artículo | To cite this article Tárraga-Mínguez, R., Vélez-Calvo, X., Sanz-Cervera, P., Pastor-Cerezuela, G. \& Fernández-Andrés, M. I. (2021). Educación inclusiva en Ecuador: perspectiva de directores, familias y evaluadores. magis, Revista Internacional de Investigación en Educación, 14, 1-21. doi: 10.11144/Javeriana.m14.eiep 


\title{
Resumen
}

El objetivo de este estudio consiste en analizar los indicadores de inclusión en una muestra de escuelas de la ciudad de Cuenca, Ecuador, y comparar las percepciones entre diferentes agentes educativos. Para ello, se les aplicó el Index for Inclusion a 19 directores(as) de centros educativos, 156 familias y 30 graduadas de titulaciones universitarias de Educación. Los resultados evidencian diferencias en la percepción del grado de inclusión en los tres grupos. Se discute el papel del sesgo de deseabilidad social en la interpretación de los resultados.

\section{Palabras clave}

Director de colegio; educación universal; familia; percepción

\begin{abstract}
The objective of this study is to analyze the inclusion indicators in a sample of schools in the city of Cuenca, and compare the perceptions among different educational agents. Nineteen directors of educational centers, 156 families and 30 graduates of university degrees in education completed the Index for Inclusion. The results show differences in the perception of the degree of inclusion in the three groups. The role of social desirability bias when interpreting the results is discussed.
\end{abstract}

\section{Keywords}

Educational administrators; universal education; family; perception 
Descripción del artículo | Article description

Este artículo presenta los resultados de una investigación elaborada en el marco del proyecto titulado Análisis de la inclusión educativa a través de indicadores de prevalencia de dificultades de aprendizaje, actitudes del profesorado y condiciones de accesibilidad en los centros de la ciudad de Cuenca (Ecuador), en la que se evaluó la percepción sobre los procesos de inclusión en centros educativos por parte de tres agentes educativos que valoraban una misma realidad: directores de las escuelas, familias de estudiantes y evaluadores externos.

\section{Introducción}

La educación inclusiva hace referencia a la presencia, participación y aprendizaje en los centros educativos de todos los estudiantes (Ainscow, Booth \& Dyson, 2006), independientemente de sus capacidades, sexo, lengua, origen étnico, estatus socioeconómico o cualquier otra condición. Se trata de un fenómeno complejo en el que se ponen en juego algunos de los conceptos más controvertidos que conciernen a la educación. Este fenómeno afecta a una serie de cuestiones relacionadas con el día a día de las escuelas, tales como la metodología educativa (De Leeuw, De Boer, Bijstra \& Minnaert, 2018) o la formación de docentes (Symeonidou, 2017). Además, tiene implicaciones económicas, ya que el logro de sus objetivos requiere de recursos personales, materiales y organizativos (Johnstone, Lazarus, Lazetic \& Nikolic, 2018; Slee, 2010). Y es también un concepto con implicaciones políticas, que se ha convertido en uno de los pilares de las leyes de varios sistemas educativos en los países occidentales (Smyth et al., 2014). En definitiva, la educación inclusiva es un concepto con claras implicaciones éticas (Reindal, 2016), que afecta a la idea amplia de educación y que tiene como objetivo primordial influir en el modelo de sociedad (Norwich, 2014).

Todas estas aristas hacen de la educación inclusiva un concepto denso, carente de una definición clara y operativa, que pueda ser fácilmente interpretada y puesta en práctica por los profesionales en las escuelas, que se define a partir de las interpretaciones (guiadas culturalmente) del corpus de literatura científica o desde las normas legislativas que hacen referencia a educación inclusiva, unas normas con notables diferencias entre regiones y que sufren cambios sustanciales a lo largo del tiempo (Reindal, 2016; Sharma, Loreman \& Macanawai, 2016).

Esta ausencia de unanimidad en la definición de la educación inclusiva hace que el concepto pueda ser interpretado por los docentes como fuente de conflictos o dilemas, generalmente relacionados con la falta de tiempo 
disponible para atender a los estudiantes con Necesidades Educativas Especiales (NEE), con falta de preparación para alcanzar los objetivos relacionados con la inclusión o con la sobrecarga de tareas que los maestros perciben (Paju, Kajamaa, Pirttimaa \& Kontu, 2018). Es por ello que, ante una misma realidad escolar, es habitual que diferentes actores perciban e interpreten los problemas relacionados con la educación inclusiva de una manera diferente.

La diferente percepción sobre educación inclusiva se ha descrito claramente al comparar las actitudes hacia la inclusión del profesorado generalista y especialista en educación especial. La investigación muestra que, en comparación con los especialistas en educación especial, el profesorado generalista es consciente de su falta de habilidades profesionales para responder a las NEE de sus alumnos, denuncian la ausencia de recursos y la sobrecarga de trabajo que les suponen las tareas relacionadas con estas, y consideran que la educación inclusiva puede derivar en una disminución de calidad de la enseñanza (Hintz, Paal, Urton, Krull, Wilbert \& Hennemann, 2015). Igualmente, se han descrito diferencias entre familias de estudiantes con NEE y profesorado en las percepciones acerca de diferentes aspectos relacionados con la inclusión que evidencian que los padres perciben la educación inclusiva como una construcción cultural, por lo que su percepción no depende de la inclusión en sí misma, sino de la construcción cultural de la realidad (Lalvani, 2013).

El objetivo principal de este estudio es analizar los indicadores de inclusión en una muestra de escuelas en Cuenca, Ecuador, y comparar las percepciones de padres y madres, directores de centro y evaluadores externos. La relevancia de este objetivo se justifica por varias razones.

En primer lugar, la colaboración entre el personal escolar y las familias es una cuestión clave para lograr una verdadera educación inclusiva en las escuelas (Haines, Gross, Blue-Banning, Francis \& Turnbull, 2015; Lyons, Thompson \& Timmons, 2016). Para lograr una colaboración efectiva, es necesario partir de un acuerdo sobre el significado de la inclusión. Por ello, la comparación de la percepción sobre la inclusión de diferentes partes interesadas puede ser el punto de partida para hacer efectiva dicha colaboración.

En segundo lugar, nuestra investigación analiza la percepción de un actor relevante para la educación inclusiva: los directores, quienes desempeñan un papel clave para promover prácticas inclusivas en sus escuelas. Investigaciones previas han demostrado consistentemente que el liderazgo de los directores es un tema clave para desarrollar estas prácticas (Ainscow, Dyson \& Weiner, 2013; Jahnukainen, 2015). Algunos factores individuales del director, como sus concepciones y puntos de vista sobre la inclusión (Graham \& Spandagou, 2011), sus percepciones de autoeficacia para afrontar los desafíos de la inclusión (Urton, Wilbert \& Hennemann, 2014) o sus actitudes e interpretación de las normas no escritas (Yan \& Sin, 2015) son 
determinantes para hacer realidad los objetivos de la inclusión. Por ello, nuestra investigación trata de analizar cuál es la percepción de la inclusión de los directores de las escuelas.

En tercer lugar, la comparación de las percepciones sobre educación inclusiva de diferentes agentes tiene una implicación importante. La información que las partes interesadas ofrecen con respecto a su percepción y actitudes hacia la inclusión puede verse influenciada por el sesgo de deseabilidad social (Lui, Sin, Yang, Forlin \& Ho, 2015; Lüke \& Grosche, 2018), ya que la educación inclusiva puede ser percibida como un asunto que lleva aparejada una actitud políticamente correcta. Ello puede generar que las personas que informan de sus actitudes y percepciones sobre la inclusión puedan sesgar sus respuestas (incluso de manera inconsciente), para ofrecer una imagen basada en opiniones socialmente aceptables.

Este sesgo de deseabilidad social y los problemas en la motivación para responder a cuestionarios escritos de autoevaluación no son exclusivos de la educación inclusiva (Faddar, Vanhoof \& De Maeyer, 2018), pero pueden influir en las respuestas de los interesados en este tema, ya que la educación inclusiva puede ser interpretada como un tema especialmente sensible, que implica respuestas y posturas correctas e incorrectas.

Para tratar de minimizar este sesgo, en la presente investigación, la educación inclusiva es evaluada por tres diferentes partes interesadas: directores y padres, quienes poseen diferentes niveles de vinculación con la escuela, lo que puede influenciar sus percepciones sobre la inclusión, y evaluadores externos, que han pasado un período prolongado de observación en las escuelas, participando de dinámicas escolares y valorando el día a día de la inclusión en estas instituciones educativas, pero que no tienen una vinculación permanente con los centros. La participación de estos evaluadores externos, que tienen un conocimiento teórico más sistemático de la educación inclusiva que los directores y las familias, puede contribuir a que la investigación tenga un juicio más independiente sobre las prácticas de inclusión en las escuelas.

Finalmente, este es el primer estudio realizado en Ecuador que compara de una manera sistemática las percepciones sobre la inclusión de diferentes agentes en los mismos centros educativos. Ecuador se encuentra actualmente inmerso en un período de profundas reformas de su sistema educativo. En los últimos años, se han aprobado varias leyes nacionales que regulan aspectos significativos de la educación inclusiva, como la Ley Orgánica de Educación Intercultural (LOEI, 31 de marzo de 2011) y la Ley Orgánica de Discapacidad (LOD, 25 de septiembre de 2012). Estas reformas educativas han implicado por primera vez en el sistema educativo ecuatoriano la introducción de los principios de participación e inclusión. Sin embargo, hasta la fecha, no existen estudios exhaustivos que evalúen 
la efectividad de estas políticas inclusivas. Esta investigación puede ser un primer paso para evaluar los resultados de las reformas educativas con respecto a la educación inclusiva en el sistema educativo ecuatoriano.

\section{Metodología}

\section{Muestra}

El presente estudio se realizó en 19 centros educativos (37,6\% públicos y $62,4 \%$ privados), ubicados en la región urbana de la ciudad de Cuenca, Ecuador, una zona que aglutina al $65,6 \%$ de la población y al $97 \%$ de la actividad económica del cantón de Cuenca, donde el porcentaje de analfabetismo es del 4,9\% y el nivel de escolaridad es de 10,9 años (Instituto Nacional de Estadística y Censos, 2010).

En el estudio participaron 19 directores(as) de centro, 156 padres y madres de estudiantes de dichos centros y 30 graduadas de titulaciones universitarias de Educación que realizaron una evaluación externa de los procesos de inclusión en estos centros escolares. Este grupo de 30 colaboradoras, formado por estudiantes de doctorado, ya había participado previamente en otras investigaciones similares a las del presente estudio como parte de su formación doctoral. Durante su proceso de formación profesional habían cursado asignaturas obligatorias sobre educación especial, el tratamiento de la diversidad y educación inclusiva. Además, para la presente investigación, asistieron a un seminario de 10 horas de duración, en el que se explicó el propósito y contenido del instrumento de evaluación, de cuyo análisis surgieron pautas para la observación durante su estancia en el centro educativo. En la tabla 1 se muestra la distribución de sexo, edad y nivel de formación de los participantes.

Tabla 1

Características de los participantes

\begin{tabular}{|c|c|c|c|}
\hline & Padres/madres & Directores(as) & Evaluadoras externos \\
\hline \multicolumn{4}{|l|}{ Sexo (porcentaje) } \\
\hline Hombre & $49(31,41 \%)$ & $9(47,37 \%)$ & 0 \\
\hline Mujer & $107(68,59 \%)$ & $10(52,63 \%)$ & $30(100 \%)$ \\
\hline Edad media (DT) & $45,75(11,06)$ & $35,76(8,20)$ & $22,37(1,71)$ \\
\hline \multicolumn{4}{|c|}{ Nivel de formación (porcentaje) } \\
\hline Bachiller & $87(55,77 \%)$ & $1(5,6 \%)$ & 0 \\
\hline Técnico(a)/tecnológico & $7(4,49 \%)$ & $2(11,1 \%)$ & 0 \\
\hline Profesor(a) o licenciado(a) & $58(37,18 \%)$ & $10(52,63 \%)$ & $30(100 \%)$ \\
\hline Especialista/máster & $4(2,56 \%)$ & $5(26,32 \%)$ & 0 \\
\hline
\end{tabular}

Fuente: elaboración propia 


\section{Instrumentos}

Se utilizó el Index for Inclusion, de Booth \& Ainscow (2002), uno de los instrumentos más empleados a nivel internacional para la evaluación de tres dimensiones relacionadas con la inclusión: la cultura, la política y las prácticas inclusivas de las instituciones educativas (Vélez-Calvo, Tárraga-Mínguez, Fernández-Andrés, Pastor-Cerezuela \& Sanz-Cervera, 2016).

Booth \& Ainscow (2002) definen la cultura inclusiva como la creación de una comunidad escolar segura, acogedora, colaborativa y estimulante, con valores inclusivos compartidos por todos sus miembros; las políticas inclusivas, como los procedimientos que persiguen que la inclusión penetre en la organización y modalidades de apoyo para mejorar el aprendizaje y la participación de todo el alumnado, y las prácticas inclusivas, como las actividades del día a día del centro que reflejan en la práctica los principios inclusivos que guían las dos dimensiones anteriores.

Las tres dimensiones del instrumento se descomponen en secciones y estas, a su vez, en indicadores, que son evaluados a través de los diferentes ítems. El instrumento original de Booth \& Ainscow (2002) constaba de 489 preguntas que abordaban un amplio abanico de cuestiones relacionadas con la educación inclusiva, lo que representaba un número excesivo para los objetivos del presente estudio. Por ello, se seleccionó un listado de 35 ítems del conjunto total de la herramienta original, correspondientes a las dimensiones y secciones indicadas en la tabla 2, ya que estas eran las más relacionadas con los objetivos de este estudio. Finalmente, se adaptaron las expresiones que fueron necesarias al contexto de Ecuador, siguiendo las directrices proporcionadas por el propio instrumento.

Una vez realizada esta adaptación, se solicitó la colaboración de cinco profesores universitarios, cuatro padres de familia de estudiantes con discapacidad, cuatro directores de centros educativos, cinco profesores de estos centros y cinco funcionarios de la coordinación zonal, quienes proporcionaron indicaciones sobre el contenido del cuestionario y sobre los ítems que podrían resultar ambiguos o confusos. Una vez recibido el feedback de estos colaboradores, se reescribieron los ítems que fueron necesarios y se conformó la versión definitiva del cuestionario que se empleó en este estudio, cuya estructura se resume en la tabla 2.

Los ítems adoptaron la forma de escala Likert, con cuatro opciones de respuesta: muy de acuerdo, de acuerdo, en desacuerdo y muy en desacuerdo, siguiendo el procedimiento empleado en diferentes estudios previos que han utilizado también el Index for Inclusion (Moliner, Sales, Ferrández \& Traver, 2011).

Los índices de fiabilidad de la prueba resultaron satisfactorios. El índice alfa de Cronbach fue de .95 en la escala de cultura inclusiva, de .92 
en la escala de políticas inclusivas y de .94 en la escala de prácticas inclusivas. Finalmente, el alfa de Cronbach para el total de ítems de la escala fue de 97.

Tabla 2

Distribución de los ítems en las dimensiones y secciones del Index for Inclusion

\begin{tabular}{l|l}
\hline Dimensión A: Crear CULTURAS inclusivas & 12 ítems \\
Sección A.2. Establecer valores inclusivos & \\
\hline Dimensión B: Elaborar POLÍTICAS inclusivas & \\
Sección B.1. Desarrollar una escuela para todos & 5 ítems \\
Sección B.2. Organizar el apoyo para atender a la diversidad & 6 ítems \\
\hline Dimensión C: Desarrollar PRÁCTICAS inclusivas & \\
Sección C.1. Orquestar el proceso de aprendizaje & 8 ítems \\
Sección C.2. Movilizar recursos & 4 ítems \\
\hline
\end{tabular}

Fuente: elaboración propia

\section{Procedimiento}

Se contactó personalmente con el equipo de dirección de los centros educativos para informar de los objetivos y características del estudio, así como para solicitar los consentimientos necesarios para llevarlo a cabo. Una vez conseguida la conformidad para realizar la investigación, las evaluadoras se desplazaban a los centros en grupos de dos o tres personas (en función del número de aulas de la escuela), y permanecían en el centro en horario lectivo durante dos semanas. Durante su estancia en los centros, las evaluadoras distribuyeron su horario para asistir a clases de todos los niveles educativos, estuvieron presentes en el patio y asistieron a reuniones de coordinación de profesorado. Para realizar tareas de observación, las evaluadoras utilizaron un diario de campo, en el que anotaban las situaciones de la jornada escolar con implicaciones para la inclusión en las relaciones entre estudiantes, profesores y profesores-estudiantes, así como aspectos relacionados con la metodología de aula, el diseño universal de aprendizaje y la existencia de recursos personales, materiales y organizativos para la educación inclusiva. Sin embargo, se instruyó a las evaluadoras para que prestaran atención en sus observaciones a otras posibles situaciones fortuitas del día a día de los centros relacionadas con la educación inclusiva que les permitieran cumplimentar los indicadores del Index for Inclusion recogidos en el instrumento de evaluación de este estudio.

Al acabar la jornada escolar, estas mismas evaluadoras se encargaban de desarrollar sesiones en las que explicaban a las familias el contenido del instrumento de evaluación y proporcionaban un ejemplar de este a las familias que libremente aceptaban colaborar en el estudio. 


\section{Resultados}

Los análisis se realizaron con el paquete estadístico SPSS, versión 24 para Windows. En primer lugar, se realizó un análisis descriptivo y una comparación de medias de cada uno de los 35 ítems del instrumento. Posteriormente, se realizó un análisis de varianza multivariado (MANOVA) para comparar las puntuaciones en las tres escalas del instrumento: crear culturas inclusivas, establecer políticas inclusivas y desarrollar prácticas inclusivas, entre los tres grupos de participantes (directores, padres y madres, y evaluadoras externas).

\section{Análisis de los ítems del Index for Inclusion}

Tabla 3

Comparación de los ítems del Index for Inclusion entre los tres grupos de participantes

\begin{tabular}{|c|c|c|c|c|}
\hline \multicolumn{2}{|c|}{ Escala/ítem } & D & $\mathbf{P}$ & $\mathbf{E}$ \\
\hline & & \multicolumn{3}{|c|}{ Media (desv. típica) } \\
\hline \multirow{12}{*}{ 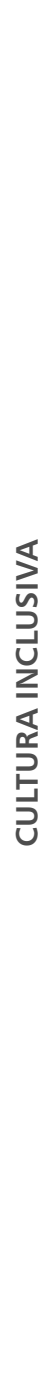 } & $\begin{array}{l}\text { El alumnado siente valorados sus logros en relación con sus propias } \\
\text { posibilidades, en vez de compararlos con el logro de los demás. }\end{array}$ & $1,53(0,51)$ & $1,76(0,84)$ & $2,57(0,90)$ \\
\hline & El alumnado está motivado para apreciar los logros de los demás. & $1,42(0,51)$ & $1,79(0,81)$ & $2,37(0,85)$ \\
\hline & $\begin{array}{l}\text { Se evita el uso de estereotipos de estudiantes a través de } \\
\text { etiquetas generales, especialmente del alumnado con } \\
\text { dificultades de aprendizaje o de otras etnias, contextos y culturas. }\end{array}$ & $1,42(0,51)$ & $1,89(0,93)$ & $2,47(0,77)$ \\
\hline & $\begin{array}{l}\text { La diversidad es considerada como un recurso rico para apoyar el } \\
\text { aprendizaje, en vez de un problema u obstáculo a evitar. }\end{array}$ & $1,26(0,45)$ & $1,79(0,86)$ & $2,83(0,70)$ \\
\hline & $\begin{array}{l}\text { Se valora de igual forma al alumnado y personal con } \\
\text { discapacidad y sin discapacidad. }\end{array}$ & $1,37(0,68)$ & $1,76(0,84)$ & $2,43(0,90)$ \\
\hline & $\begin{array}{l}\text { Se considera a todos los miembros de la escuela como personas } \\
\text { que aprenden y al mismo tiempo enseñan. }\end{array}$ & $1,16(0,38)$ & $1,80(0,82)$ & $2,43(0,86)$ \\
\hline & $\begin{array}{l}\text { Todo el personal de la escuela se siente valorado y apoyado, } \\
\text { independientemente de su cargo o función que desempeña } \\
\text { en el centro. }\end{array}$ & $1,26(0,45)$ & $1,85(0,88)$ & $2,30(0,84)$ \\
\hline & El personal evita humillar a algún estudiante o colega en particular. & $1,16(0,38)$ & $1,78(0,84)$ & $2,03(0,81)$ \\
\hline & $\begin{array}{l}\text { La escuela intenta disminuir toda discriminación institucional, } \\
\text { en relación con la edad, la "raza", el origen social o cultural, la } \\
\text { orientación sexual, el género, la discapacidad o el logro de } \\
\text { los estudiantes. }\end{array}$ & $1,05(0,23)$ & $1,76(0,85)$ & $2,13(0,68)$ \\
\hline & $\begin{array}{l}\text { El profesorado evita ver la deficiencia como la única causa de las } \\
\text { dificultades que experimentan en la escuela los estudiantes con } \\
\text { discapacidad. }\end{array}$ & $1,37(0,60)$ & $1,81(0,84)$ & $2,60(0,86)$ \\
\hline & Se desafían las opiniones estereotipadas sobre la perfección física. & $1,47(0,77)$ & $1,89(0,89)$ & $2,03(0,78)$ \\
\hline & $\begin{array}{l}\text { El personal intenta contrarrestar las actitudes estereotipadas } \\
\text { hacia las personas con discapacidad (por ejemplo, que son } \\
\text { incapaces de tener relaciones, que merecen compasión o que son } \\
\text { luchadores heroicos contra la adversidad). }\end{array}$ & $1,42(0,51)$ & $1,87(0,87)$ & $2,63(0,81)$ \\
\hline
\end{tabular}


Hay una preocupación por la accesibilidad de los edificios y las áreas del centro en todos los aspectos, incluyendo las aulas, los pasillos, los baños, los jardines, las áreas de juego, el comedor o cafetería y las exposiciones.

Se han tomado medidas para ir mejorando la accesibilidad al centro y a sus dependencias como parte del proyecto de la escuela.

La escuela se preocupa por conocer la legislación existente en el país en relación con la accesibilidad de las personas con discapacidad y el diseño universal.

La escuela tiene un programa de acogida para el alumnado.

Media (desv. típica)

El alumnado nuevo tiene claro a quién tiene que acudir si experimenta dificultades.

Todo el personal ofrece igualdad de oportunidades educativas a las personas con discapacidad.

Los estudiantes etiquetados con "necesidades educativas especiales" no son un grupo homogéneo sino que tienen diferentes intereses, conocimientos y habilidades.

Los profesionales de apoyo se incorporan al aula, en lugar de "retirar" de ella a determinados alumnos para que reciban apoyo pedagógico.

Las prácticas de evaluación y el apoyo pedagógico están integrados dentro de la política general de la escuela sobre la inclusión.

Las adaptaciones curriculares individualizadas sirven para mejorar las estrategias de enseñanza y aprendizaje para todos los alumnos.

Los informes de evaluación de los alumnos con "necesidades educativas especiales" especifican el apoyo necesario para maximizar su participación en el currículo común y en la comunidad.

Las clases se preparan teniendo en cuenta el lenguaje que los alumnos utilizan fuera del centro educativo.

\begin{tabular}{l|l|l}
$1,42(0,61)$ & $1,95(0,90)$ & 3,07 \\
$(0,79)$
\end{tabular}

\begin{tabular}{l|l|l}
$1,44(0,51)$ & $1,95(0,92)$ & $2,73(0,64)$
\end{tabular}

$1,58(0,61) \quad 1,88(0,88) \quad 2,60(0,72)$

\begin{tabular}{|l|l|l|} 
& & \\
$1,56(0,62)$ & $1,94(0,88)$ & $2,50(0,68)$
\end{tabular}

$1,47(0,51) \quad 1,84(0,80) \quad 2,73(0,79)$

$1,53(0,70) \quad 1,78(0,79) \quad 2,53(0,68)$

El personal reconoce el esfuerzo físico que realizan algunos estudiantes con discapacidad o enfermedades crónicas para completar las tareas, y el cansancio que ello puede causarles. El personal reconoce el esfuerzo mental que emplean algunos estudiantes, por ejemplo, porque tienen que leer en los labios o utilizar ayudas visuales.

El profesor reconoce el tiempo suplementario que necesitan y emplean algunos estudiantes con discapacidad para utilizar los instrumentos en trabajos prácticos o en manualidades.

Se utilizan estrategias de evaluación diferentes, de forma que se permita a todos los estudiantes mostrar sus habilidades.

Se hace un seguimiento de los logros de diferentes grupos de estudiantes (niños, niñas, estudiantes de pueblos originarios, estudiantes con discapacidad), para detectar y abordar dificultades específicas. 


\begin{tabular}{|c|c|c|c|c|}
\hline \multirow{2}{*}{\multicolumn{2}{|c|}{ Escala/ítem }} & D & $\mathbf{P}$ & $\mathbf{E}$ \\
\hline & & \multicolumn{3}{|c|}{ Media (desv. típica) } \\
\hline \multirow{6}{*}{ 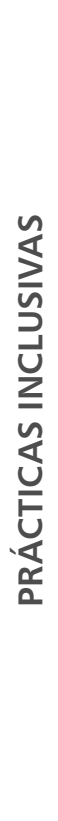 } & $\begin{array}{l}\text { Los docentes están abiertos a los comentarios de otros } \\
\text { colegas sobre cuestiones tales como la claridad del lenguaje } \\
\text { de instrucción, o la participación de los estudiantes en las } \\
\text { actividades programadas. }\end{array}$ & $1,37(0,60)$ & $1,79(0,83)$ & $2,57(0,86)$ \\
\hline & $\begin{array}{l}\text { El profesorado modifica su docencia en función de las } \\
\text { recomendaciones recibidas de sus colegas. }\end{array}$ & $1,42(0,61)$ & $1,81(0,82)$ & $2,77(0,82)$ \\
\hline & $\begin{array}{l}\text { Los recursos de apoyo se dirigen a prevenir las barreras al } \\
\text { aprendizaje y la participación y a disminuir la clasificación o } \\
\text { etiquetaje del alumnado. }\end{array}$ & $1,58(0,77)$ & $1,90(0,91)$ & $2,93(0,79)$ \\
\hline & $\begin{array}{l}\text { El profesorado con habilidades y conocimientos específicos } \\
\text { ofrece su ayuda a los demás. }\end{array}$ & $1,37(0,76)$ & $1,81(0,87)$ & $2,60(0,89)$ \\
\hline & $\begin{array}{l}\text { En la escuela hay un clima que posibilite proponer alternativas } \\
\text { al resto de profesorado en relación con las dificultades } \\
\text { del alumnado. }\end{array}$ & $1,47(0,61)$ & $1,95(0,92)$ & $2,70(0,79)$ \\
\hline & $\begin{array}{l}\text { Si los hay, el personal de los centros de educación especial de } \\
\text { la zona participa con el personal de las escuelas comunes en el } \\
\text { intercambio de experiencias. }\end{array}$ & $2,22(1,11)$ & $2,20(1,10)$ & $3,30(0,84$ \\
\hline
\end{tabular}

D: directores(as), P: padres y madres, E: evaluadoras

Fuente: elaboración propia

La comparación de medias en todos los ítems indica que los directores(as) tienen la puntuación más baja de los tres grupos (lo que sugiere una visión más positiva de los procesos de educación inclusiva) y las evaluadoras externas tienen la puntuación más alta de los tres grupos (lo que sugiere una visión más pesimista acerca de estos procesos). Los padres y madres se sitúan en una situación intermedia entre los dos restantes grupos en todos los ítems.

\section{Diferencias grupales en las tres dimensiones del Index for Inclusion}

EI MANOVA realizado en las tres dimensiones reveló diferencias estadísticamente significativas entre los tres grupos de participantes en todas las subescalas evaluadas (lambda de Wilk $(\lambda)=.744 ; F(6,400)=10,65$; $\left.p<.001 ; \eta_{p}^{2}=.138\right)$.

Como muestra la tabla 4, en todos los casos, las evaluadoras externas valoraron la cultura, las políticas y las prácticas de la escuela como menos inclusivas en comparación con los otros dos grupos. Del mismo modo, los padres y madres evaluaron las dimensiones de la cultura y las políticas como menos inclusivas en comparación con los directores de las escuelas. No hubo diferencias en la dimensión de las prácticas inclusivas entre los padres y madres de estudiantes y los directores de las escuelas. 
Tabla 4

Comparación de las escalas del Index for Inclusion entre los tres grupos de participantes

\begin{tabular}{|c|c|c|c|c|c|c|}
\hline & \multicolumn{3}{|c|}{ Grupos } & \multirow{3}{*}{$F_{(1,77)}$} & \multirow{3}{*}{$\eta_{\mathrm{p}}^{2}$} & \multirow{3}{*}{$\begin{array}{l}\text { Comparación } \\
\text { entre grupos }\end{array}$} \\
\hline & \multirow{2}{*}{$\begin{array}{c}\text { D } \\
\text { M } \\
(\mathrm{DT})\end{array}$} & \multirow{2}{*}{$\begin{array}{c}\mathbf{P} \\
\mathbf{M} \\
(\mathrm{DT})\end{array}$} & \multirow{2}{*}{$\begin{array}{c}\mathrm{E} \\
\mathrm{M} \\
(\mathrm{DT})\end{array}$} & & & \\
\hline & & & & & & \\
\hline Crear culturas inclusivas & $\begin{array}{c}15,9 \\
(3,68)\end{array}$ & $\begin{array}{l}21,66 \\
(8,44)\end{array}$ & $\begin{array}{l}28,80 \\
(7,32)\end{array}$ & $16,60 * *$ & .141 & $\begin{array}{c}\mathrm{D}<\mathrm{P}, \mathrm{E} \\
\mathrm{P}<\mathrm{E}\end{array}$ \\
\hline Establecer políticas inclusivas & $\begin{array}{c}16,11 \\
(4,95)\end{array}$ & $\begin{array}{l}20,73 \\
(7,68)\end{array}$ & $\begin{array}{l}30,57 \\
(5,75)\end{array}$ & $29,71 * *$ & .227 & $\begin{array}{c}\mathrm{D}<\mathrm{P}, \mathrm{E} \\
\mathrm{P}<\mathrm{E}\end{array}$ \\
\hline Desarrollar prácticas inclusivas & $\begin{array}{l}17,79 \\
(6,66)\end{array}$ & $\begin{array}{l}22,24 \\
(8,52)\end{array}$ & $\begin{array}{l}32,23 \\
(7,16)\end{array}$ & $23,48 * *$ & .189 & $D, P<E$ \\
\hline
\end{tabular}

** $p<.016$ corrección de Bonferroni. D: directores(as), P: padres y madres, E: evaluadoras

Fuente: elaboración propia

Para calcular las puntuaciones que aparecen en la tabla, se realizó para cada participante un sumatorio de las puntuaciones en la escala Likert (entre 1 y 4) de los ítems correspondientes a cada escala y posteriormente se calculó la media y desviación típica de las puntuaciones de cada participante. Dado que las escalas de culturas y prácticas inclusivas constaban de 12 ítems, la puntuación máxima en estas escalas era de 48, mientras que en la escala de políticas inclusivas, que constaba de 11 ítems, la puntuación máxima era de 44. Las puntuaciones están calculadas en orden inverso, es decir, una puntuación más baja significa una mejor percepción de la cultura, políticas y prácticas inclusivas, mientras que una puntuación mayor significa una peor percepción de estos constructos.

\section{Discusión}

La percepción de los procesos de inclusión educativa en las escuelas públicas y privadas de la ciudad de Cuenca, Ecuador, es diferente para los tres agentes participantes en la presente investigación: directores, padres y madres de estudiantes y evaluadoras externas. Este resultado sugiere que la dinámica inclusiva de las escuelas no es percibida como una realidad nítidamente imparcial, sino que la percepción de la inclusión depende, al menos en parte, del rol del observador.

En el presente estudio, el grupo de evaluadoras externas, integrado por estudiantes de doctorado con formación académica en inclusión, percibió los espacios escolares como significativamente menos inclusivos que los padres y madres de estudiantes y los directores de centro. Los padres y madres se sitúan en una posición intermedia entre los otros dos grupos, ya 
que valoran la realidad escolar como significativamente más inclusiva que las evaluadoras externas, pero tienen una percepción menos optimista con respecto a la inclusión que los directores de los centros en las dimensiones de cultura y políticas inclusivas. Y finalmente, los directores de los centros escolares valoran la misma realidad escolar como significativamente más inclusiva que los otros dos grupos.

Esta diferencia en la valoración de una misma realidad escolar puede responder, al menos, a tres factores relevantes: los conocimientos sobre inclusión del observador; su vínculo con la escuela y, por tanto, la presencia del sesgo de deseabilidad social, y el grado de abstracción expresado en los ítems dentro de las dimensiones evaluadas.

En primer lugar, con respecto a los conocimientos sobre inclusión, las diferencias en cuanto a formación reglada en esta temática pueden explicar las discrepancias entre las evaluadoras externas con los otros dos grupos evaluados. Tal y como muestran investigaciones previas, el conocimiento sobre algunos aspectos de la educación inclusiva es un tema relevante en la generación de actitudes hacia la inclusión (Lui et al., 2015).

Por un lado, en esta investigación, se ha obtenido que las evaluadoras externas tienen una mayor formación en el ámbito de la inclusión que los padres. Por otro lado, esta misma diferencia en cuanto a la capacitación en inclusión puede ser intuida cuando comparamos la formación de las evaluadoras externas con la de los directores de las escuelas. En este sentido, Vélez-Calvo et al. (2016) concluyeron que los cursos que tratan la inclusión dentro de los programas de formación del profesorado en Ecuador incluyen únicamente un promedio de 2,17 asignaturas por titulación, una situación que no conduce a una adecuada preparación en educación inclusiva y que es notablemente inferior a la que poseían las evaluadoras externas del presente estudio.

Aplicado a los resultados obtenidos, las condiciones en cuanto a formación inclusiva en el profesorado ecuatoriano pueden haber influido en la percepción de la inclusión por parte de los directores. La escasa formación en inclusión habitualmente genera dificultades para diferenciar entre integración e inclusión (Woodcock \& Hardy, 2017), y dificulta la posibilidad de que la inclusión se sitúe como el motor para el cambio en la cultura, las políticas y las prácticas de los centros, de modo que se acojan a todos los estudiantes y se responda adecuadamente a sus necesidades de aprendizaje (Ainscow et al., 2013).

Por su parte, es probable que el mayor nivel de formación y comprensión sobre la inclusión educativa de las evaluadoras externas haya generado un mayor nivel de exigencia en la valoración de la cultura, las políticas y las prácticas educativas. Esta mayor exigencia puede ser la responsable de 
que su percepción de la realidad educativa fuera significativamente menos inclusiva con respecto a los otros dos grupos, quienes probablemente no fueron tan estrictos al evaluar los procesos de inclusión en sus centros.

El segundo factor que puede dar cuenta de las diferencias en la percepción de los tres grupos de participantes de una misma realidad escolar es el de la vinculación de los participantes con el centro que están valorando. Las evaluadoras externas carecían de una relación previa con los centros analizados en este estudio, por lo que su valoración estaba exenta de posibles prejuicios y sesgos derivados de una experiencia previa con ellos. Por el contrario, tanto el grupo de padres y madres como el de directores formaban parte del propio centro que estaban valorando, lo que inevitablemente puede haber afectado su valoración con respecto a los procesos de inclusión.

En el caso concreto de los directores, es posible que puedan haber percibido que el cuestionario estaba evaluando su propio trabajo como profesionales, es decir, podrían haberse sentido como sujetos investigados y, por lo tanto, condicionados a mostrar una versión edulcorada e idealizada de la realidad, respondiendo más en el sentido de lo que consideran que deberían ser sus escuelas, en lugar de lo que realmente son.

En cuanto a la relación con la escuela, el grupo de padres y madres se situaba en una posición intermedia con respecto a los otros dos grupos, ya que, aunque probablemente son el grupo más interesado en que los centros educativos adquieran un carácter inclusivo (al ser sus hijos e hijas quienes son educados en esos centros), no son un grupo que verdaderamente forme parte del personal del centro. Por ello, probablemente cuando responden el cuestionario, lo hacen pensando que son ellos quienes están evaluando al centro, pero no se sienten evaluados, como es posible que ocurra en el caso de los directores.

Esta gradación en la vinculación con la escuela en los tres grupos participantes en la investigación -1) evaluadores que observan "desde afuera"; 2) directores que valoran "desde adentro", y 3) las familias, ubicadas en una posición intermedia, con intereses legítimos en la escuela, pero sin formar parte realmente del núcleo del centro- puede ser una de las causas de que los procesos de inclusión se perciban de manera diferente por los tres grupos. Este grado de pertenencia o cercanía al centro puede hacer que el sesgo de deseabilidad social haya influido de manera diferente en los diferentes grupos.

Finalmente, el tercer factor que puede ayudar a interpretar las diferencias en los resultados de los tres grupos es el grado de abstracción de los ítems del instrumento empleado. Las dimensiones de cultura y políticas inclusivas tienen un contenido más abstracto que la dimensión de prácticas 
inclusivas, que se refiere a aspectos más tangibles y directamente observables de las experiencias escolares cotidianas.

Esta diferencia en el grado de abstracción de las escalas puede explicar por qué las diferencias entre padres y directores fueron significativas en las dimensiones de cultura y políticas inclusivas, dimensiones más abstractas, más teóricas y, por tanto, difíciles de relacionar con los hechos concretos que acontecen en el día a día de las escuelas; mientras que no hubo diferencias en la dimensión de prácticas educativas, una dimensión que hace referencia a aspectos más concretos, ligados al día a día de los centros y, por tanto, en la que es más difícil que difieran los agentes que observan y participan cotidianamente en los centros. transformación encaminado a mejorar la educación de todos en la escuela. Este proceso requiere de figuras que ejerzan un liderazgo verdaderamente concienciado de la necesidad de desarrollar prácticas inclusivas (Graham \& Spandagou, 2011). Los directores de centros educativos ocupan un lugar idóneo dentro de la organización de los centros para responsabilizarse de ese liderazgo (Yan \& Sin, 2015), pero los resultados de esta investigación no son alentadores en este sentido, y constatan que es necesario articular medidas para revertir esta situación de falta de conciencia en cuanto a la educación inclusiva. Entre las posibles medidas para poder aumentar la concienciación de los directores de los centros educativos en el ámbito de la inclusión, estaría la inversión en formación inicial y continua de estos directores, ya que esta preparación afectará su propia comprensión de la inclusión, así como de la gestión, el cuidado y la promoción de la diversidad. Esta formación debe superar la antigua concepción restrictiva de la inclusión como un ámbito meramente limitado a la educación especial (Symeonidou, 2017), y debería hacer hincapié en aspectos como el liderazgo para la transformación de los centros educativos, la concienciación de toda 
la organización de la necesidad de autoevaluar y mejorar los procesos de inclusión y el desarrollo de estrategias didácticas y organizativas que aseguren que en los centros educativos se respeta, de manera efectiva, el derecho de todos a la educación, independientemente de su sexo, capacidad, lengua, origen socioeconómico o cualquier otra condición.

Otra conclusión que se deriva de los resultados del presente estudio es que se evidencia la importancia de aplicar procesos de autoevaluación para monitorear el progreso hacia prácticas educativas más inclusivas (Forlin, 2004). Se trata de procesos construidos "desde abajo", que evalúan las condiciones reales de inclusión desde la escuela y que planifican acciones específicamente dirigidas a mejorar los procesos inclusivos para responder a esa realidad escolar particular. De lo contrario, existe el riesgo de que la inclusión se refleje meramente en las leyes diseñadas "desde arriba" (FuIlan, 2007), y que su aplicación no llegue verdaderamente a las aulas. Aunque Ecuador ha tenido un escenario legal que favorece la inclusión escolar desde 2011, si la comunidad escolar no percibe la inclusión como una meta, estas reformas educativas pueden convertirse en meras normas ornamentales, que tan solo afectan de manera superficial la realidad escolar, pero que no transforman verdaderamente las prácticas educativas. Para evitar esta situación, la inclusión debe considerarse como un principio organizado y práctico, en el que la comunidad escolar debe desarrollar la capacidad de construirlo progresivamente.

Las diferentes percepciones de las dimensiones de la inclusión nos permiten apreciar que es posible que los participantes de la presente investigación entiendan la filosofía de la educación inclusiva de manera diferente. La educación inclusiva no es un concepto sencillo de definir, y probablemente su interpretación no es plenamente compartida por los miembros de la comunidad escolar (Engelbrecht, Oswald \& Forlin, 2006), lo que supone una cuestión relevante para que una institución educativa pueda convertirse en una escuela realmente inclusiva (Dyson, 2001; Stockall \& Gartin, 2002).

Finalmente, consideramos necesario remarcar que el estudio de las percepciones acerca de la educación inclusiva no necesariamente se corresponde con una medición o evaluación en términos psicométricos de la realidad inclusiva de los centros educativos. En el presente estudio nos hemos aproximado a esta realidad a través de la percepción de diferentes agentes. Debe tenerse en cuenta que una percepción puede estar influenciada por sesgos, deseos, motivaciones, formación previa en la materia, etc., lo que representa una temática muy rica que nos ayuda a entender algo más un fenómeno tan complejo como la educación inclusiva, pero que no debemos confundir con una medición supuestamente monolítica y exacta de la educación inclusiva. 


\section{Limitaciones del estudio}

El presente trabajo tiene algunas limitaciones, que deben tenerse en cuenta al interpretar los resultados. En primer lugar, las evaluadoras externas pasaron un tiempo relativamente corto (dos semanas) en las escuelas, una situación que podría limitar la validez de sus observaciones, ya que este tiempo supone un período breve, aunque consideramos que es suficiente para analizar los centros. La opción de limitar los períodos de observación a únicamente dos semanas se justifica en que uno de los objetivos del estudio era precisamente contar con una visión externa a los centros, cuya valoración de la inclusión estuviera exenta del sesgo inherente a los agentes que forman verdaderamente parte de la institución escolar, y que, por tanto, tienen un bagaje previo que puede orientar las respuestas debido a sesgos, como el de deseabilidad social.

Además, la presente investigación se basa únicamente en métodos cuantitativos. No se emplearon métodos cualitativos que pudieran haber contribuido a un análisis más profundo de algunos de los centros participantes. Esta cuestión queda pendiente para futuros estudios.

\section{Futuras líneas de investigación}

Este estudio abre algunas líneas interesantes para futuras investigaciones:

En primer lugar, los resultados sugieren la necesidad de acometer en el escenario escolar ecuatoriano el proceso de investigación-acción recomendado por el Index for Inclusion (Booth \& Ainscow, 2002), tal y como se ha llevado a cabo con éxito en escuelas de otros sistemas educativos (Céspedes \& Muñoz, 2014; Sales, Traver \& García, 2011). Este tipo de estudios, hasta ahora inédito en Ecuador, muestra cómo son los propios centros educativos quienes autoevalúan su cultura, su política y sus prácticas educativas inclusivas, en un proceso iniciado por la organización de un grupo de coordinación que trabaja con los miembros de la comunidad; analiza la escuela para identificar las barreras de aprendizaje y participación; define las prioridades que permiten proponer etapas hacia la implementación de acciones de mejora concretas, y evalúa de manera continua el progreso de las medidas implementadas.

A este respecto, consideramos que sería apropiado, además, incluir en este proceso de autoevaluación a los propios estudiantes de las escuelas, quienes a menudo han estado ausentes en estos equipos de evaluación (McMaster, 2013). Numerosas investigaciones con resultados prometedores han evidenciado que, cuando todos los actores de la escuela reman en una misma dirección, los procesos de inclusión y la calidad escolar mejoran (Haines et al., 2015; Lyons et al., 2016), por lo que consideramos que es 
relevante incluir en futuros estudios también el análisis de las percepciones de los estudiantes.

Otra línea de trabajo interesante abierta a partir de la presente investigación sería el análisis de las percepciones de las familias de los estudiantes acerca de la inclusión, diferenciando entre familias de niños con y sin NEE. En el presente estudio no hemos podido realizar este análisis. Sin embargo, creemos que sería relevante analizar si existen diferencias en la percepción de los procesos de inclusión de ambos grupos de familias, tal y como ocurre en otros sistemas educativos (Stevens \& Worf, 2018).

Finalmente, consideramos necesario recalcar que, debido a las recientes reformas legislativas en materia de educación, el sistema educativo ecuatoriano se encuentra actualmente en un momento histórico que puede derivar en dos posibilidades antagónicas: por un lado, es posible, y sería deseable, que las reformas del marco normativo conduzcan de manera efectiva a una transformación educativa real y tangible en el día a día de los centros y que los transforme para, poco a poco, ser centros verdaderamente inclusivos. Para ello, creemos que es necesaria la participación de todos los agentes educativos: directores, profesorado, familias, formadores de profesorado e investigadores del ámbito de la educación. Con la participación de todos, es posible que se produzcan cambios reales en las escuelas. Pero, por otro lado, es posible que si los cambios legales no van acompañados del suficiente esfuerzo en la investigación y la transformación de las escuelas desde las propias escuelas, corramos el riesgo de que las reformas queden en papel mojado y sirvan únicamente para maquillar la realidad educativa sin penetrar verdaderamente en la cotidianidad de los centros.

\section{Sobre los autores}

Raúl Tárraga-Mínguez es profesor de la Universidad de Valencia, España. Su línea de investigación se centra en el análisis de la percepción social de las necesidades educativas especiales y la formación del profesorado de educación especial.

Ximena Vélez-Calvo es profesora de la Universidad del Azuay, Ecuador. Su línea de investigación se centra en la educación especial y la propuesta de mejoras del sistema educativo.

Pilar Sanz-Cervera es profesora de la Universidad de Valencia, España. Su línea de investigación se centra en el estudio del trastorno del espectro autista y sus implicaciones educativas.

Gemma Pastor-Cerezuela es profesora de la Universidad de Valencia, España. Su línea de investigación se centra en el estudio del trastorno del espectro autista y sus implicaciones educativas. 
María Inmaculada Fernández-Andrés es profesora de la Universidad de Valencia, España. Su línea de investigación gira en torno al estudio de diferentes aspectos relacionados con la educación de niños con trastornos del neurodesarrollo.

\section{Referencias}

Ainscow, M., Booth, T. \& Dyson, A. (2006). Improving Schools, Developing Inclusion. Londres: Routledge.

Ainscow, M., Dyson, A. \& Weiner, S. (2013). The Tasks of Leadership: From Exclusion to Inclusion. Manchester: University of Manchester, Centre for Equity in Education.

Faddar, J., Vanhoof, J. \& De Maeyer, S. (2018). School self-evaluation: Self-perception or self-deception? The impact of motivation and socially desirable responding on self-evaluation results. Journal School Effectiveness and School Improvement, 29(4), 660-678. doi: 10.1080/09243453.2018.1504802

Forlin, C. (2004). Promoting inclusivity in Western Australian schools. International Journal of Inclusive Education, 8(2), 185-202. doi: 10.1080/136031 1032000158042

Fullan, M. G. (2007). The New Meaning of Educational Change. Nueva York: Teachers College.

Graham, L. J. \& Spandagou. I. (2011). From vision to reality: Views of primary school principals on inclusive education in New South Wales, Australia. Disability \& Society, 26(2), 223-237. doi: 10.1080/09687599.2011.544062

Haines, S. J., Gross, J. M., Blue-Banning, M., Francis, G. L. \& Turnbull, A. P. (2015). Fostering family-school and community-school partnerships in inclusive schools: Using practice as a guide. Research and Practice for Persons with Severe Disabilities, 40(3), 227-239. doi: 10.1177/1540796915594141

Hintz, A. M., Paal, M., Urton, K., Krull, J., Wilbert, J. \& Hennemann, T. (2015). Teachers' perceptions of opportunities and threats concerning inclusive schooling in Germany at an early stage of inclusion: Analyses of a mixed methodology approach. Journal of Cognitive Education and Psychology, 14(3), 357-374. doi: 10.1891/1945-8959.14.3.357 
Instituto Nacional de Estadística y Censos (INEC). (2010). Censo de Población y Vivienda. Recuperado de https://www.ecuadorencifras.gob.ec/base-dedatos-censo-de-poblacion-y-vivienda-2010

Jahnukainen, M. (2015). Inclusion, integration, or what? A comparative study of the school principals' perceptions of inclusive and special education in Finland and in Alberta, Canada. Disability \& Society, 30(1), 59-72. doi: 10.1080/09687599.2014.982788

Johnstone, C., Lazarus, S., Lazetic, P. \& Nikolic, G. (2018). Resourcing inclusion: Introducing finance perspectives to inclusive education policy rhetoric. Prospects, 1-21. doi: 10.1007/s11125-018-9432-2

Lalvani, P. (2013). Land of misfit toys: Mothers' perceptions of educational environments for their children with Down syndrome. International Journal of Inclusive Education, 17(5), 435-448. doi: 10.1080/13603116.2012.683047

Ley Orgánica de Discapacidades (LOD). Registro Oficial del Gobierno del Ecuador N. ${ }^{\circ}$ 796, 25 de septiembre de 2012. Recuperado de http://www.consejodis capacidades.gob.ec/wp-content/uploads/downloads/2014/02/ley_organi ca_discapacidades.pdf

Ley Orgánica de Educación Intercultural (LOEI). Registro Oficial del Gobierno del Ecuador N. ${ }^{\circ}$ 417, 31 de marzo de 2011. Recuperado de https://educacion. gob.ec/ley-organica-de-educacion-intercultural-loei/

Lui, M., Sin, K. F., Yang, L., Forlin, C. \& Ho, F. C. (2015). Knowledge and perceived social norm predict parents' attitudes towards inclusive education. International Journal of Inclusive Education, 19(10), 1052-1067. doi: 10.1080/13603116.2015.1037866

Lüke, T. \& Grosche, M. (2018). What do I think about inclusive education? It depends on who is asking. Experimental evidence for a social desirability bias in attitudes towards inclusion. International Journal of Inclusive Education, 22(1), 38-53. doi: 10.1080/13603116.2017.1348548

Lyons, W. E., Thompson, S. A. \& Timmons, V. (2016). "We are inclusive. We are a team. Let's just do it": Commitment, collective efficacy, and agency in four inclusive schools. International Journal of Inclusive Education, 20(8), 889-907. doi: 10.1080/13603116.2015.1122841

McMaster, C. (2013). The inclusive practices tools: Trying to take a short cut to inclusion. New Zealand Journal of Teacher's Work, 10(2), 220-230.

Moliner, O., Sales, A., Ferrández, R. \& Traver, J. (2011). Inclusive cultures, policies and practices in Spanish compulsory secondary education schools: Teachers' perceptions in ordinary and specific teaching contexts. International Journal of Inclusive Education, 15(5), 557-572. doi: 10.1080/13603110903165158

Norwich, B. (2014). Recognising value tensions that underlie problems in inclusive education. Cambridge Journal of Education, 44(4), 495-510. doi: 10.1080/0305764X.2014.963027

Paju, B., Kajamaa, A., Pirttimaa, R. \& Kontu, E. (2018). Contradictions as drivers for improving inclusion in teaching pupils with special educational needs. Journal of Education and Learning, 7(3), 11-22. doi: 10.5539/jel.v7n3p11

Reindal, S. M. (2016). Discussing inclusive education: An inquiry into different interpretations and a search for ethical aspects of inclusion using the 
capabilities approach. European Journal of Special Needs Education, 31(1), 1-12. doi: 10.1080/08856257.2015.1087123

Sales, A., Traver, J. A. \& García, R. (2011). Action research as a school-based strategy in intercultural professional development for teachers. Teaching and Teacher Education, 27(5), 911-919. doi: 10.1016/j.tate.2011.03.002

Sharma, U., Loreman, T. \& Macanawai, S. (2016). Factors contributing to the implementation of inclusive education in Pacific Island countries. International Journal of Inclusive Education, 20(4), 397-412. doi: 10.1080/1360 3116.2015 .1081636

Slee, R. (2010). Political economy, inclusive education and teacher education. En C. Forlin (Ed.), Teacher Education for Inclusion (pp. 39-48). Nueva York: Routledge.

Smyth, F. et al. (2014). Inclusive education in progress: Policy evolution in four European countries. European Journal of Special Needs Education, 29(4), 433-445. doi: 10.1080/08856257.2014.922797

Stevens, L. \& Wurf, G. (2018). Perceptions of inclusive education: A mixed methods investigation of parental attitudes in three Australian primary schools. International Journal of Inclusive Education, 1-15. doi: 10.1080/136 03116.2018.1464068

Stockall, N. \& Gartin, B. (2002). The nature of inclusion in a blue ribbon school: A revelatory case. Exceptionality, 10(3), 171-188. doi: 10.1207/ S15327035EX1003_2

Symeonidou, S. (2017). Initial teacher education for inclusion: A review of the literature. Disability \& Society, 32(3), 401-422. doi: 10.1080/096875 99.2017 .1298992

Urton, K., Wilbert, J. \& Hennemann, T. (2014). Attitudes towards inclusion and self-efficacy of principals and teachers. Learning Disabilities-A Contemporary Journal, 12(2), 151-168.

Vélez-Calvo, X., Tárraga-Mínguez, R., Fernández-Andrés, M. I., Pastor-Cerezuela, G. \& Sanz-Cervera, P. (2016). Formación inicial de maestros en educación inclusiva, una comparación entre Ecuador y España. Revista de Educación Inclusiva, 9(3), 75-94.

Woodcock, S. \& Hardy, I. (2017). Beyond the binary: Rethinking teachers' understandings of and engagement with inclusion. International Journal of Inclusive Education, 21(6), 667-686. doi: 10.1080/13603116.2016.1251501

Yan, Z. \& Sin, K. F. (2015). Exploring the intentions and practices of principals regarding inclusive education: An application of the Theory of Planned Behaviour. Cambridge Journal of Education, 45(2), 205-221. doi: 10.1080/0305764X.2014.934203 